\title{
Estimation of Arterial Carbon Dioxide Based on End-Tidal Gas Pressure and Oxygen Saturation
}

\author{
Raisa Rentola ${ }^{1}{ }^{\oplus}$, Johanna Hästbacka ${ }^{1}$, Erkki Heinonen ${ }^{2}$, Per H. Rosenberg ${ }^{3}$, Tom Häggblom ${ }^{2}$ \\ and Markus B. Skrifvars 1,4,* \\ 1 Division of Intensive Care, Department of Anaesthesiology, Intensive Care and Pain Medicine, \\ Helsinki University Hospital and University of Helsinki, 00290 Helsinki, Finland; \\ raisa.rentola@hus.fi (R.R.); johanna.hastbacka@hus.fi (J.H.) \\ 2 Clinical Care Solutions, Anaesthesia and Respiratory Care, 00510 Helsinki, Finland; \\ erkki.heinonen@ge.com (E.H.); tom.haggblom@ge.com (T.H.) \\ 3 Division of Anaesthesia, Department of Anaesthesiology, Intensive Care and Pain Medicine, \\ Helsinki University Hospital and University of Helsinki, 00290 Helsinki, Finland; per.rosenberg@fimnet.fi \\ 4 Department of Emergency Care and Services, University of Helsinki and Helsinki University Hospital, \\ 00290 Helsinki, Finland \\ * Correspondence: markus.skrifvars@hus.fi; Tel.: +358-504272424
}

Received: 27 June 2018; Accepted: 13 September 2018; Published: 19 September 2018

\begin{abstract}
Arterial blood gas (ABG) analysis is the traditional method for measuring the partial pressure of carbon dioxide. In mechanically ventilated patients a continuous noninvasive monitoring of carbon dioxide would obviously be attractive. In the current study, we present a novel formula for noninvasive estimation of arterial carbon dioxide. Eighty-one datasets were collected from 19 anesthetized and mechanically ventilated pigs. Eleven animals were mechanically ventilated without interventions. In the remaining eight pigs the partial pressure of carbon dioxide was manipulated. The new formula (Formula 1) is $\mathrm{PaCO}_{2}=\mathrm{PETCO}_{2}+\mathrm{k}\left(\mathrm{PETO}_{2}-\mathrm{PaO}_{2}\right)$ where $\mathrm{PaO}_{2}$ was calculated from the oxygen saturation. We tested the agreements of this novel formula and compared it to a traditional method using the baseline $\mathrm{PaCO}_{2}-\mathrm{ETCO}_{2}$ gap added to subsequently measured, end-tidal carbon dioxide levels (Formula 2). The mean difference between $\mathrm{PaCO}_{2}$ and calculated carbon dioxide (Formula 1) was $0.16 \mathrm{kPa}\left( \pm \mathrm{SE}\right.$ 1.17). The mean difference between $\mathrm{PaCO}_{2}$ and carbon dioxide with Formula 2 was $0.66 \mathrm{kPa}( \pm \mathrm{SE} 0.18)$. With a mixed linear model excluding cases with cardiorespiratory collapse, there was a significant difference between formulae $(p<0.001)$, as well as significant interaction between formulae and time $(p<0.001)$. In this preliminary animal study, this novel formula appears to have a reasonable agreement with $\mathrm{PaCO}_{2}$ values measured with ABG analysis, but needs further validation in human patients.
\end{abstract}

Keywords: arterial carbon dioxide; mechanical ventilation; noninvasive measurement; blood gas analysis

\section{Introduction}

Mechanical ventilation is one of the most common practices in emergency and critical care settings. The primary objective is to achieve and maintain sufficient oxygen supply for organs and an adequate clearance of carbon dioxide $\left(\mathrm{CO}_{2}\right)$ from the body [1]. Considering $\mathrm{CO}_{2}$, both hypocapnia and hypercapnia should be avoided [2]. Frequent control of the arterial partial pressure of carbon dioxide $\left(\mathrm{PaCO}_{2}\right)$ is needed especially for patients with brain injury because carbon dioxide dilates the cerebral blood vessels and may, therefore, increase intracranial pressure [3]. Hypocapnia, on the other hand, causes cerebral vasoconstriction and may lead to regional cerebral ischemia, and has been shown to 
worsen outcomes in patients with traumatic brain injury $[4,5]$. Nonetheless, unintentional hypocapnia commonly occurs in clinical practice [6].

Arterial blood gas $(\mathrm{ABG})$ analysis is the reference method for monitoring adequate ventilation in mechanically ventilated patients [4]. Consequently, $\mathrm{ABG}$ analysis is the most frequent laboratory test in the intensive care unit (ICU) [7]. The average number of ABG samples varies; in a study from our department, eight samples were taken during the first $24 \mathrm{~h}$ in patients treated following cardiac arrest [8]. ABG analysis is also difficult or impossible in some situations, such as in the prehospital setting and during patient transportation [9].

A noninvasive method for estimating arterial $\mathrm{CO}_{2}$ would have obvious implications for clinical practice. A commonly used strategy, under stable conditions, is to calculate the gap between end-tidal $\mathrm{CO}_{2}\left(\mathrm{ETCO}_{2}\right)$ and $\mathrm{PaCO}_{2}$ under the assumption that the gap remains constant, and use the continuous $\mathrm{ETCO}_{2}$ value for arterial $\mathrm{CO}_{2}$ estimation over time. However, because the gap may change, this method is likely to become inaccurate with time. In this experimental animal study, we tested a novel formula for calculating arterial carbon dioxide partial pressure. The formula utilizes noninvasive patient gas parameters readily available in ventilated patients and could provide an alternative way to determine $\mathrm{PaCO}_{2}$ value without $A B G$ analysis. We hypothesized that this formula would outperform traditional end-tidal $\mathrm{CO}_{2}\left(\mathrm{ETCO}_{2}\right)$-based $\mathrm{PaCO}_{2}$ prediction, also in situations involving concomitant changes in ventilation and perfusion.

\section{Materials and Methods}

The current study consists of an analysis of data collected during a series of animal experiments undertaken at the Research and Development Unit of the Helsinki University Hospital, Helsinki, Finland between September 2015 and September 2016. The animal experiments were a part of a developmental project where a novel adaptive ventilation control system was applied on traditional pressure- (PCV) and volume-controlled (VCV) mechanical ventilation and on pressure support (CPAP-PSV) of spontaneously triggered breathing. The developmental project was conducted for internal purposes only, and it will not be published in a scientific journal. The study protocol was approved by the National Animal Experiment Board (ESAVI/1801/04.10.07/2015; Hämeenlinna, Finland) and by the Hospital Board of Helsinki University Hospital (17 June 2015).

\subsection{Study Subjects}

Nineteen Landrace pigs of both genders were used for the experiments. The average weight of the pigs was $28.7 \pm 10.1 \mathrm{~kg}$ (range 15.5-47.0 kg). All animals were fasted overnight but had free access to water. Twenty minutes prior to testing, the animals were premedicated with intramuscular medetomidine $(80 \mu \mathrm{g} / \mathrm{kg})$ and ketamine $(10 \mathrm{mg} / \mathrm{kg})$. An ear vein was cannulated, and anesthesia was induced using bolus doses of propofol $(4-5 \mathrm{mg} / \mathrm{kg})$. After tracheal intubation, the pigs were mechanically ventilated using either volume control ventilation (VCV), pressure control ventilation $(\mathrm{PCV})$, or continuous positive airway pressure combined with pressure support ventilation (CPAP + PSV) mode. A five-lead electrocardiogram and peripheral capillary oxygen saturation through a pulse oximeter were recorded continuously. Respiratory gases (fraction inspired carbon dioxide $\left[\mathrm{FiCO}_{2}\right], \mathrm{ETCO}_{2}$, fraction inspired oxygen $\left[\mathrm{FIO}_{2}\right]$ and end tidal oxygen $\left[\mathrm{ETO}_{2}\right]$ ) were measured with an S/5 Anaesthesia Monitor (GE Healthcare, Helsinki, Finland) [10]. Blood gases were measured using an epoc ${ }^{\circledR}$ Blood Analysis System (Alere, Waltham, MA, USA).

Anesthesia was maintained with sevoflurane, and analgesia was provided by boluses of fentanyl. Depth of anesthesia was monitored using EntropyTM in addition to visual observation. The femoral artery was cannulated for blood pressure monitoring and arterial blood sampling, and blood pressure was measured invasively. A central venous catheter was inserted in some cases for monitoring of central venous pressure, infusion of intravenous fluids and medication, and for creating an air embolism as described below. After completing the test, the animals were euthanized with a bolus 
of potassium chloride. Baseline characteristics, physiological parameters, and data on mechanical ventilation are shown in Supplementary Tables S1 and S2.

\subsection{Experimental Procedures}

The first set of ABG measurements was taken after induction of anesthesia under stable hemodynamical conditions in normoventilated animals. In two animals, the testing included different depths of anesthesia and awakening with spontaneous breathing. In one animal, the procedures included mildly elevated abdominal pressure and awakening. In 10 animals (pigs 9-19), the only intervention undertaken was elevation of intra-abdominal pressure by inflating carbon dioxide into the abdominal cavity to a pressure of $15 \mathrm{mmHg}$. In eight animals (pigs 1-8), various procedures were undertaken in an attempt to manipulate the arterial content of carbon dioxide.

These included:

- Inducing metabolic acidosis with an infusion of lactate (30-32 $\mathrm{mmol})$ followed by infusion of $1 \mathrm{~mL} / \mathrm{kg}$ sodium bicarbonate to increase the $\mathrm{CO}_{2}$ content;

- Experimental air embolism with the injection of $60 \mathrm{~mL}$ of air into the central vein;

- Lung injury with the injection of $0.9 \%$ sodium chloride endobronchially;

- Endobronchial ventilation with blocking of the left bronchus with the inflated balloon of a Swan-Ganz catheter balloon acting as a bronchial blocker;

- Trendelenburg position.

Considering further ABG samples, a 15 min stabilization period was allowed after each intervention in order to reach hemodynamic stability before sampling. The only exception was in a case where an air embolus was injected: in a situation of rapidly deteriorating hemodynamics, stabilization was not possible and the sample was taken without a stabilization period. The outline of the experimental protocol is shown in the Supplemental Figure S1a.

\subsection{Estimation of Arterial $\mathrm{CO}_{2}$ Partial Pressure}

Synchronously with the measurement of $\mathrm{ABG}$ we obtained data on respiratory gases, i.e., $\mathrm{FICO}_{2}$, $\mathrm{ETCO}_{2}, \mathrm{FIO}_{2}$, and $\mathrm{ETO}_{2}$, and used these in a formula for the estimation of $\mathrm{PaCO}_{2}$.

The novel formula (Formula 1) used for prediction of $\mathrm{PaCO}_{2}$ is defined as

$$
\mathrm{PaCO}_{2}=\mathrm{PETCO}_{2}+\mathrm{k}\left(\mathrm{PETO}_{2}-\mathrm{PaO}_{2}\right) .
$$

The relationship factor $\mathrm{k}$ is defined by fitting unpublished datapoints for the minimal difference between the blood gas measured $\mathrm{PaCO}_{2}$ and the Formula 1 calculated value. $\mathrm{PETCO}_{2}$ and $\mathrm{PETO}_{2}$ are the respective measured dry gas end-tidal volume \% values converted to body temperature and pressure saturated partial pressures. For this purpose, we utilized one datapoint from each of 190 emergency patients breathing room air and of 43 ICU ventilated patients recently treated in our university hospital. This fitting gave the value $\mathrm{k}=0.035$ for samples when $\mathrm{ETO}_{2} \leq 60 \mathrm{kPa}$ and $\mathrm{k}=0.01$ when $\mathrm{ETO}_{2}>60 \mathrm{kPa} . \mathrm{PaCO}_{2}$ and $\mathrm{PaO}_{2}$ are the arterial $\mathrm{CO}_{2}$ and $\mathrm{O}_{2}$ partial pressures, respectively, and $\mathrm{PETCO}_{2}$ and $\mathrm{PETO}_{2}$ are the end-tidal $\mathrm{CO}_{2}$ and $\mathrm{O}_{2}$ pressures. The estimation of $\mathrm{PaO}_{2}$ was based on values from the oxygen dissociation curve assuming a normal $\mathrm{pH}$ level and is presented in Supplementary Materials Table S3 [11]. Despite differences in the oxygen dissociation curve between humans and pigs, we did not use a porcine-specific method for measuring hemoglobin saturation, possibly overestimating the $\mathrm{PaO}_{2}$ level [12].

In addition to Formula 1, we used another method to estimate $\mathrm{PaCO}_{2}$. First, the difference between measured $\mathrm{PaCO}_{2}$ and $\mathrm{ETCO}_{2}$ was calculated from the first available ABG and the corresponding capnography value. This difference was added to the subsequent $\mathrm{ETCO}_{2}$ values at the times when subsequent $\mathrm{ABG}$ samples were taken (Formula 2: $\mathrm{PaCO}_{2}=\mathrm{ETCO}_{2}+\left(\mathrm{PaCO}_{2}-\mathrm{ETCO}_{2}\right)$ ). We compared this method to our novel formula (Formula 1). 
Formulae:

$$
\begin{array}{ll}
\text { Formula } 1 & \mathrm{PaCO}_{2}=\mathrm{PETCO}_{2}+\mathrm{k}\left(\mathrm{PETO}_{2}-\mathrm{PaO}_{2}\right) \\
\text { Formula } 2 & \mathrm{PaCO}_{2}=\mathrm{ETCO}_{2}+\left(\mathrm{PaCO}_{2}-\mathrm{ETCO}_{2}\right) .
\end{array}
$$

\subsection{Statistical Analyses}

Categorical variables are expressed as a number and percentage. Normally and non-normally distributed continuous variables are expressed as mean $( \pm \mathrm{SD})$ and median (range), respectively. To demonstrate the agreement between the estimated and measured values of blood $\mathrm{PaCO}_{2}$, we created Bland-Altman plots [13] with 95\% confidence intervals. Mean differences between the measured $\mathrm{PaCO}_{2}$ and both formulae with the limits of agreement and their respective $95 \%$ confidence intervals were calculated. The standard deviations of the differences with their standard errors (SE) were calculated. Within-subject and between-subject variances (WSW and BSV) and intra-class correlations $(\tau)$ were calculated for both formulae as well as the repeatability coefficients. The data were not normally distributed (Kolmogorov-Smirnov test, $p$ value $<0.001$ ). The normality of the distribution of the differences between the measured and estimated values were tested using the Kolmogorov-Smirnov test ( $p$ values 0.218 and 0.138 ). The two formulae were compared, regarding the difference between the estimated and actual $\mathrm{PaCO}_{2}$ value over time, with a mixed linear model, with subjects treated as a random effect and method, time, and their interaction as fixed effects. For the Bland-Altman analysis including the bias with \pm SE and the limits of agreement with $95 \%$ confidence intervals we used the freely available software created by Olofsen et al. [14].

Other analyses were performed using Statistical Package for Social Sciences version 24 software (SPSS Inc., Chicago, IL, USA), SAS version 9.4 (SAS Institute Inc., Cary, NC, USA), and SPSS for Windows (Version 22.0, IBM, Armonk, NY, USA).

\section{Results}

In total, 81 datasets were collected from 19 pigs. Baseline characteristics and data on mechanical ventilation are shown in the Supplementary Tables S2 and S3.

\subsection{Difference between Estimated and Measured Values}

In our study, we had three clear outliers. In two cases, mean arterial pressures (MAPs) were below $60 \mathrm{mmHg}$, and in one case, the MAP was decreasing rapidly and reached $25 \mathrm{mmHg}$ two minutes after arterial blood sampling. The mean difference between measured and calculated (Formula 1) $\mathrm{PaCO}_{2}$ was $0.15 \mathrm{kPa}$ (SE \pm 0.17 ). The $\mathrm{SD}$ of the differences was 1.26 ( $\mathrm{SE} \pm 0.1$ ). Formula 1 overestimated $\mathrm{PaCO}_{2}$ in $42 \%$ of cases and underestimated $\mathrm{PaCO}_{2}$ in the remaining $58 \%$. Excluding extreme outliers, the mean difference (Formula 1) was $0.055 \mathrm{kPa}$ (SD 0.44; $\mathrm{SE} \pm 0.05$ ). The mean difference between measured and calculated (Formula 1) $\mathrm{PaCO}_{2}$ before interventions was $-0.06 \mathrm{kPa}$, (SD 0.49, $\mathrm{SE} \pm 0.09$ ). The mean difference for Formula 2 was $0.66 \mathrm{kPa}(\mathrm{SE} \pm 0.18)$ and the $\mathrm{SD}$ of these differences was $1.3 \mathrm{kPa}$ (SE \pm 0.1 ). Excluding extreme outliers, the mean difference (Formula 2) was $0.42 \mathrm{kPa}$, and the $\mathrm{SD}$ of these differences was $0.44 \mathrm{kPa}$ (SE \pm 0.57 ). Formula 2 underestimated $\mathrm{PaCO}_{2}$ in $87 \%$ of cases and overestimated $\mathrm{PaCO}_{2}$ in the remaining 13\%. The Bland-Altman plots with limits of agreement and their 95\% confidence intervals demonstrating the agreement between $\mathrm{PaCO}_{2}$ (Formula 1) and measured $\mathrm{PaCO}_{2}$ and between $\mathrm{PaCO}_{2}$ (Formula 2) and measured $\mathrm{PaCO}_{2}$ are presented in Figure 1a,b. 


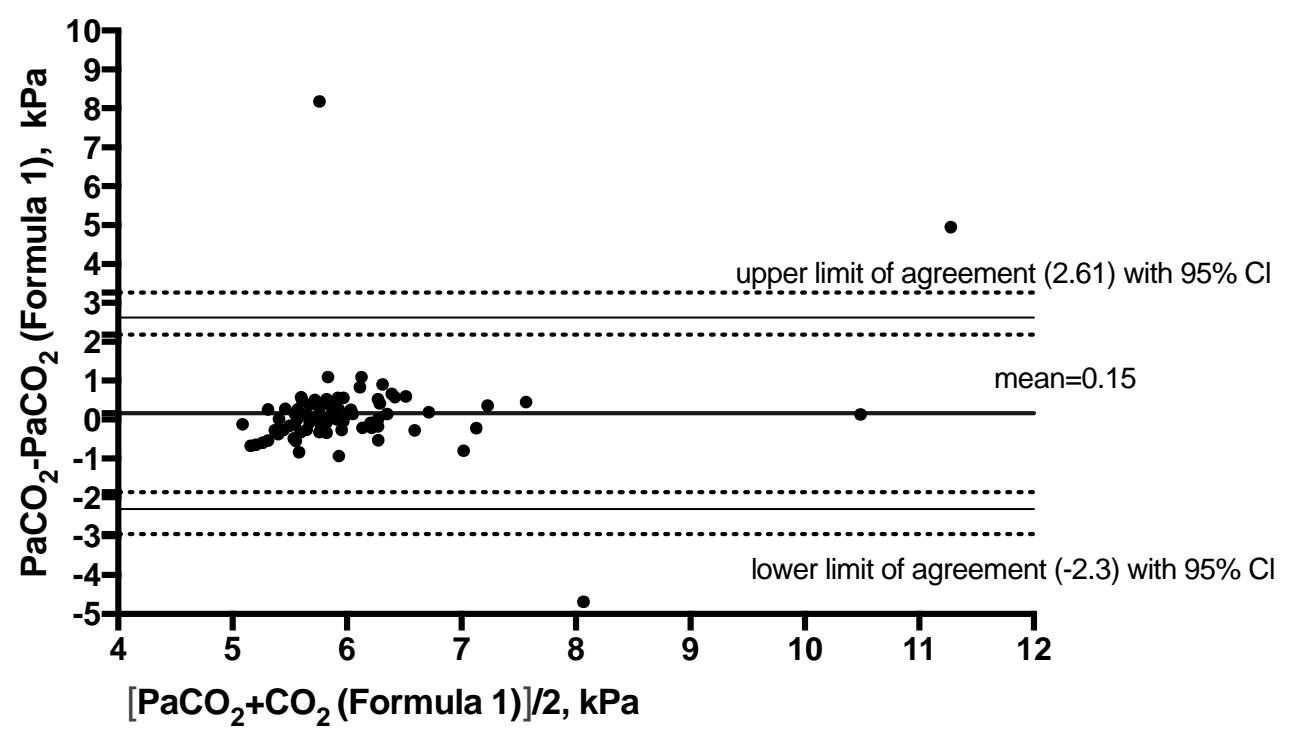

(a)

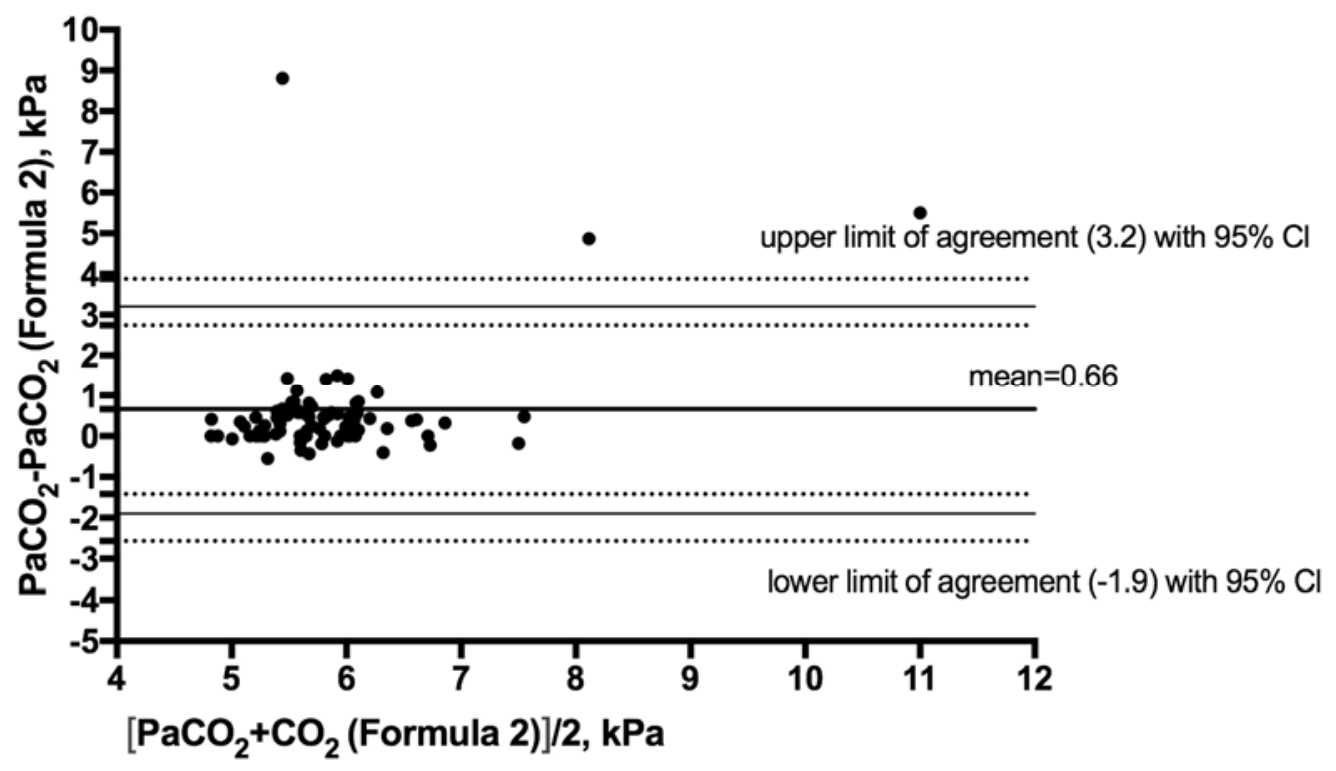

(b)

Figure 1. Bland-Altman plots with $95 \%$ limits of agreement with $95 \%$ confidence intervals (CIs) demonstrating (a) the difference between the arterial partial pressure of carbon dioxide $\left(\mathrm{PaCO}_{2}\right.$ ) (Formula 1) and measured $\mathrm{PaCO}_{2}$, and (b) the difference between $\mathrm{PaCO}_{2}$ (Formula 2) and measured $\mathrm{PaCO}_{2}$.

With linear mixed-model analysis including all values, there were no statistically significant differences between the two methods for estimating $\mathrm{PaCO}_{2}(p=0.057)$, but there was significant interaction between methods and time $(p=0.014)$. In a model excluding outliers, the difference between the methods was statistically significant $(p<0.001)$, and there was significant interaction between methods and time $(p<0.001)$. The differences between $\mathrm{PaCO}_{2}$ and $\mathrm{PaCO}_{2}$ estimated using Formulae 1 and 2 over time points are presented in Figure 2a,b. 


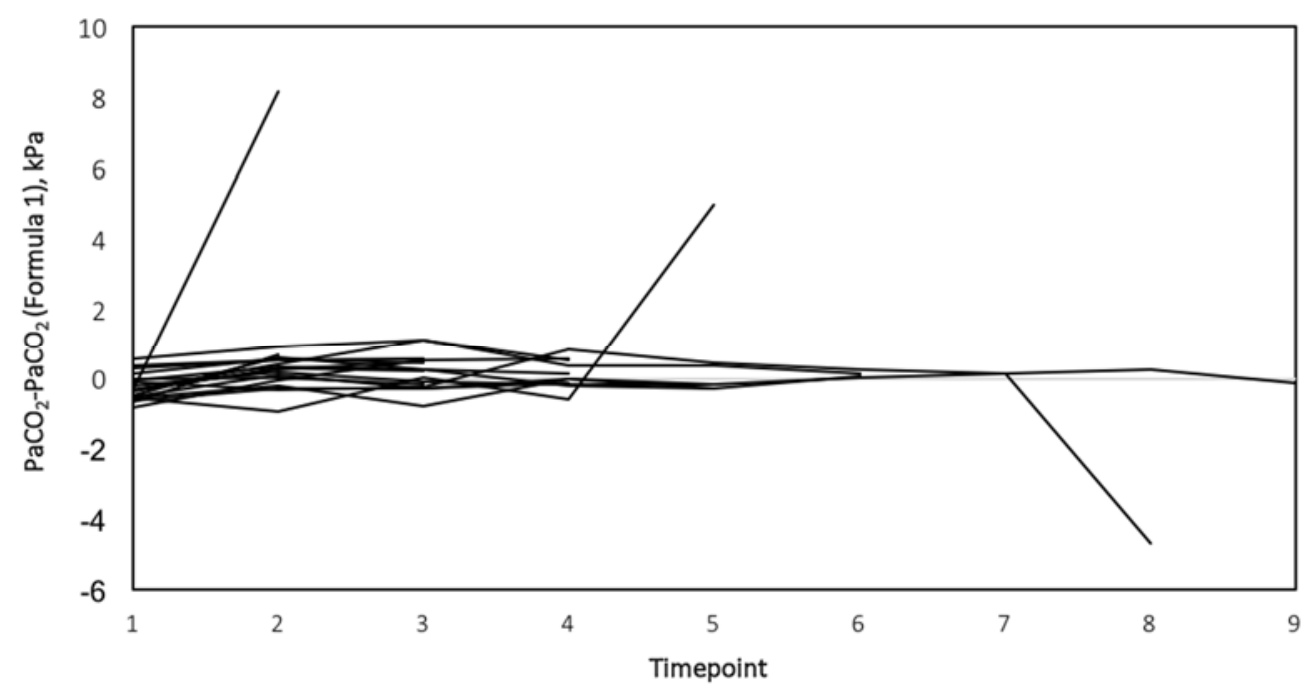

(a)

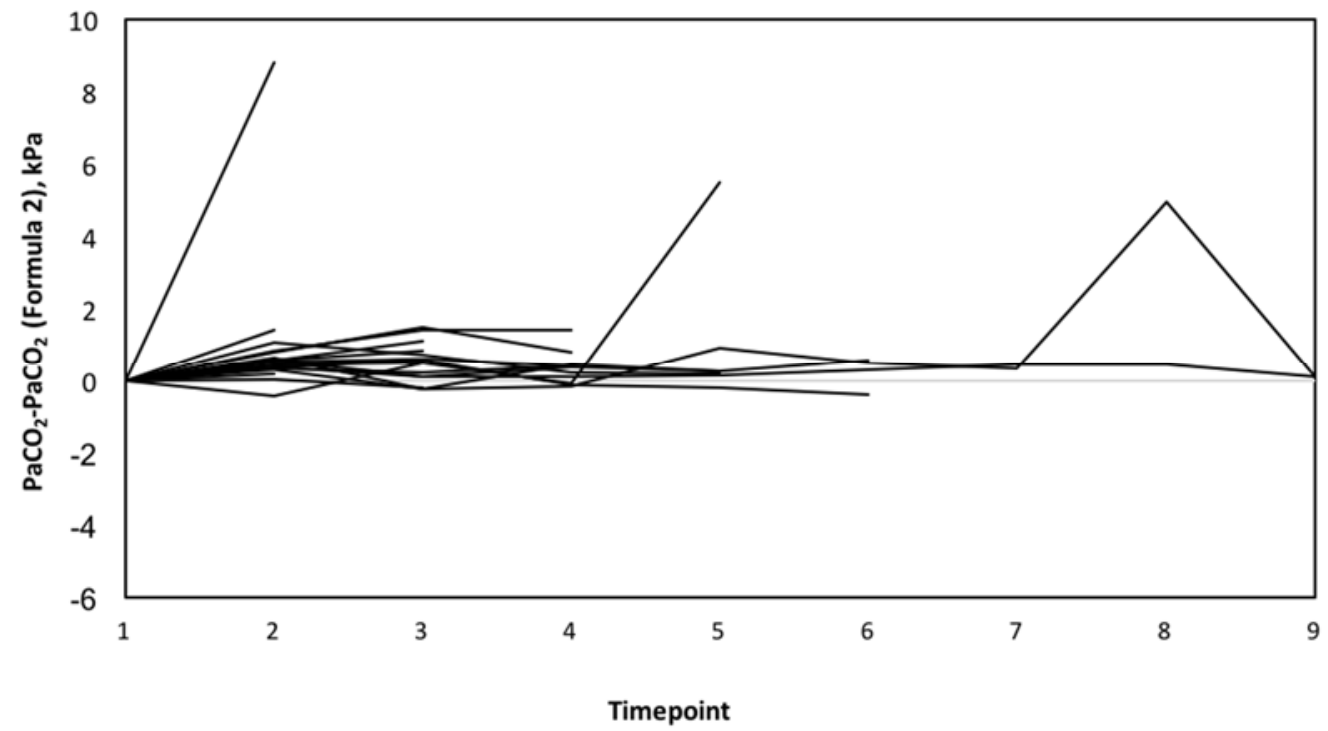

(b)

Figure 2. (a) The differences between estimated $\mathrm{PaCO}_{2}$ (Formula 1) and measured $\mathrm{CO}_{2}$ values at each time point. (b) The differences between estimated $\mathrm{PaCO}_{2}$ (Formula 2) and measured $\mathrm{CO}_{2}$ values at each time point.

\subsection{Within-Subject and Between-Subject Variance and Intra-Class Correlation}

The within-subject variances (WSV) for Formula 1 and Formula 2 were 1.43 (SE \pm 0.26 ) and 1.48 (SE \pm 0.27 ), respectively, and the between-subject variances were 0.16 ( $\mathrm{SE} \pm 0.18$ ) and 0.21 (SE \pm 0.20 ), respectively. The intra-class correlations ( $\tau=$ ratio of BSV and total variance) for Formula 1 and Formula 2 were 0.1 (SE \pm 0.1 , Spearman's $\varrho 0.31, \mathrm{SE} \pm 0.12$ ) and 0.13 (SE \pm 0.1 , Spearman's $\varrho 0.05, \mathrm{SE} \pm 0.15$ ), respectively. The differences for Formula 1 and Formula 2 in terms of individual measurements regarding each animal and according to the interventions are presented in Figure $3 \mathrm{a}, \mathrm{b}$. 


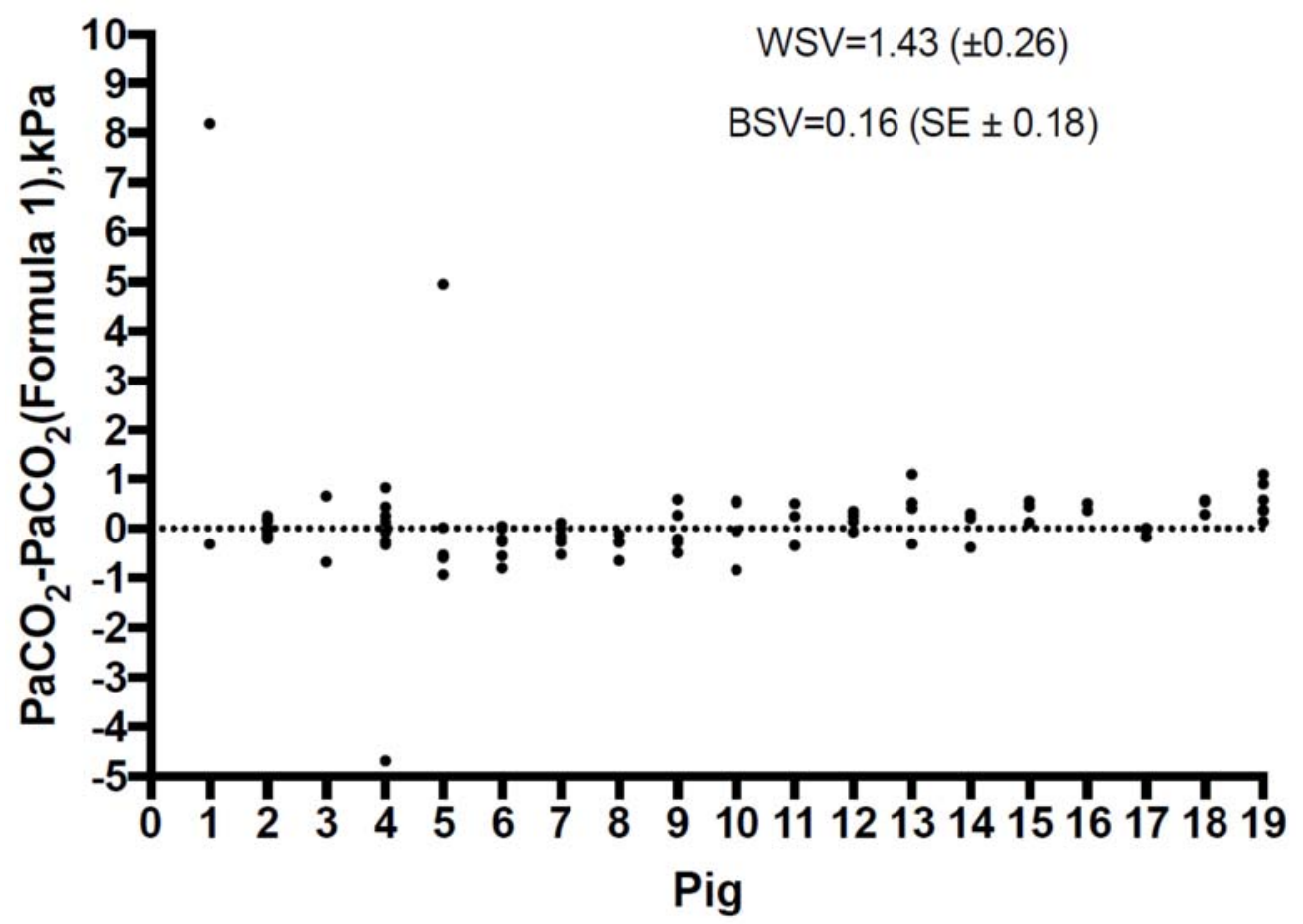

(a)

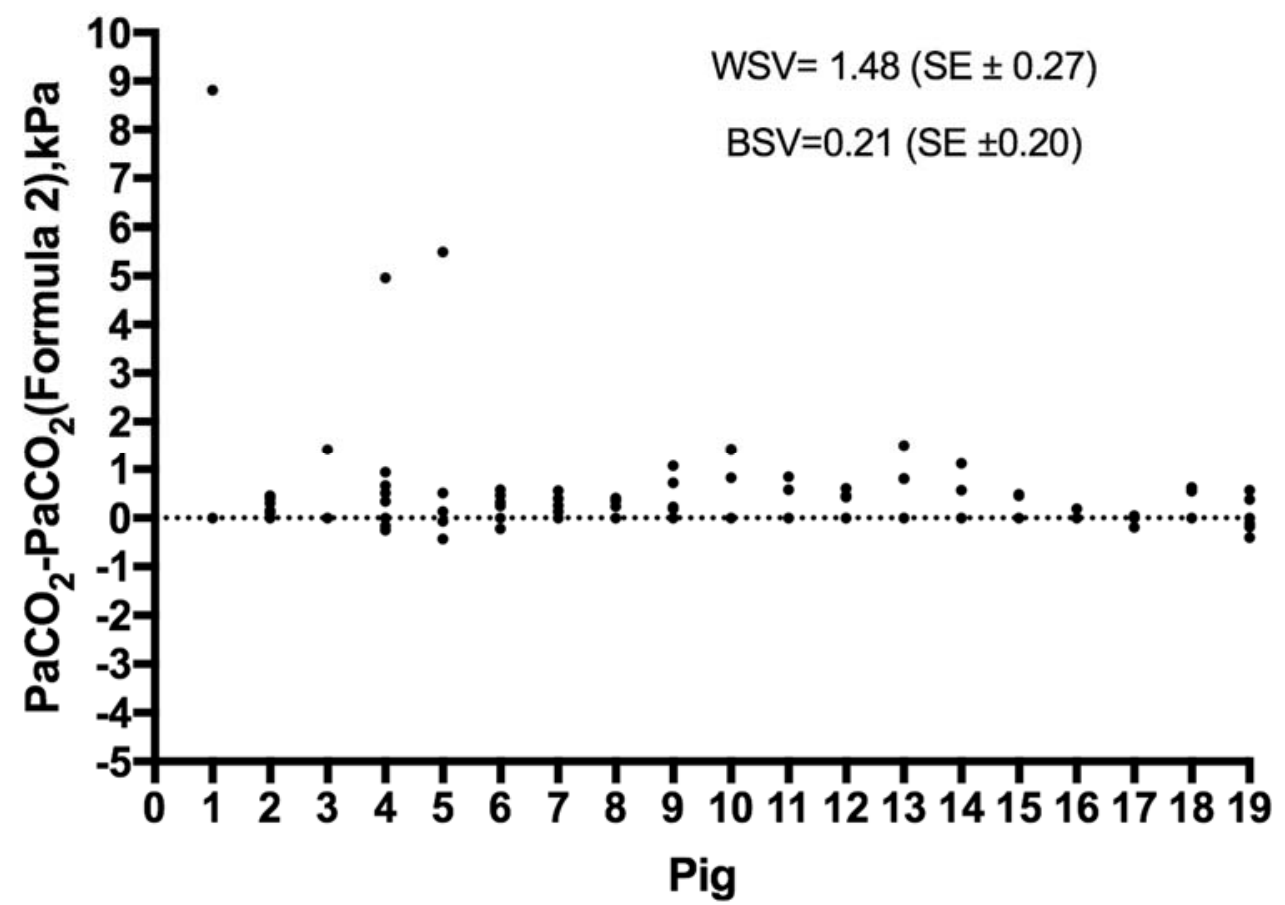

(b)

Figure 3. (a) The difference between $\mathrm{PaCO}_{2}$ (Formula 1) and measured $\mathrm{PaCO}_{2}$ for each animal. (b) The difference between $\mathrm{PaCO}_{2}$ (Formula 2) and measured $\mathrm{PaCO}_{2}$ for each animal. WSV, within-subject variance; $\mathrm{BSV}$, between-subject variance.

\subsection{Performance in Animals with Metabolic Acidosis}

The difference between $\mathrm{PaCO}_{2}$ and estimated $\mathrm{PaCO}_{2}$ by Formula 1 was largest in two pigs when metabolic acidosis was simulated (mean differences 4.25 and $1.4 \mathrm{kPa}$ ). The smallest difference was found in the pig ventilated in the Trendelenburg position (mean difference $0.21 \mathrm{kPa}$ ). 


\subsection{Performance in Animals under Stable Conditions and in Comparison with the End-Tidal $\mathrm{CO}_{2}$}

The mean difference between $\mathrm{PaCO}_{2}$ and estimated $\mathrm{PaCO}_{2}$ by Formula 1 in animals with $\mathrm{CO}_{2}$ insufflation as the only intervention (9-19) was $0.24 \mathrm{kPa}$ (SD 0.5; SE \pm 0.08 ). The corresponding mean difference for Formula 2 in these animals was $0.4 \mathrm{kPa}$ (SD 0.54; $\mathrm{SE} \pm 0.09$ ). The Bland-Altman plots with 95\% confidence intervals demonstrating the agreement between $\mathrm{PCO}_{2}$ (Formula 1) and measured $\mathrm{PaCO}_{2}$ and between $\mathrm{PaCO}_{2}$ (Formula 2) and measured $\mathrm{PaCO}_{2}$ in animals 9-19 are presented in Supplementary Material Figure S2a,b.

The mean difference between measured $\mathrm{PaCO}_{2}$ and end-tidal $\mathrm{CO}_{2}$ was $1.1 \mathrm{kPa}(\mathrm{SD} 1.3 ; \mathrm{SE} \pm 0.14$ ). The difference between end-tidal $\mathrm{CO}_{2}$ and $\mathrm{PaCO}_{2}$ over time is shown in the Supplementary Materials in Figure S3, and the Bland-Altman plot with $95 \%$ confidence intervals demonstrating the agreement between $\mathrm{PaCO}_{2}$ and $\mathrm{ETCO}_{2}$ is presented in the Supplementary Materials in Figure S4.

In an additional analysis of 26 measurements obtained prior to any intervention aiming at modifying carbon dioxide, the mean difference between the estimated $\mathrm{PaCO}_{2}$ with Formula 1 was $0.25 \mathrm{kPa}$ (SD 0.4; $\mathrm{SE} \pm 0.08$ ), The corresponding difference for $\mathrm{ETCO}_{2}$ and $\mathrm{PaCO}_{2}$ was $0.83 \mathrm{kPa}$ (SD 0.5; $\mathrm{SE} \pm 0.1$. The corresponding Bland-Altman plots with $95 \%$ confidence intervals are presented in the Supplementary Material Figure S5a,b.

\section{Discussion}

In this study, we tested the agreement of two noninvasive methods to estimate $\mathrm{PaCO}_{2}$. We found that our novel formula, which uses information from respiratory gas measurements available during mechanical ventilation, appears more accurate in a hemodynamically stable state than the commonly used $\mathrm{ETCO}_{2}$ gap method. After controlled validation in clinical studies, this novel formula may have clinical implications in cases where $\mathrm{PaCO}_{2}$ control is important but where ABG analysis is difficult, such as during patient transport. To the best of our knowledge, no studies on the estimation of $\mathrm{PaCO}_{2}$ using readily available respiratory gas values and oxygen saturation have been published.

In previous studies the acceptable range of agreement has been set at $1 \mathrm{kPa}$ between different methods of estimating $\mathrm{PaCO}_{2}$ [15]. Importantly, the clinically significant difference may vary depending on the situation; e.g., for neurosurgical patients with critically elevated intracerebral pressure, changes of more than $0.5 \mathrm{kPa}$ in $\mathrm{PaCO}_{2}$ values may be extremely significant. One key point of the novel formula is the definition of the factor $\mathrm{k}$. It has been developed from data in patients who were breathing room air, so it is likely that additional data from subjects receiving supplemental oxygen may refine this value further, especially in relationship to increased oxygen pressures.

Previous studies regarding a correlation between end-tidal carbon dioxide $\left(\mathrm{ETCO}_{2}\right)$ and $\mathrm{PaCO}_{2}$ have shown conflicting results. On one hand, Razi and colleagues found a strong correlation between $\mathrm{ETCO}_{2}$ and $\mathrm{PaCO}_{2}$ in 87 critically ill mechanically ventilated patients [16]. On the other hand, Kerr and colleagues, in a study on head trauma patients, concluded that the clinical validity of estimation of $\mathrm{PaCO}_{2}$ with $\mathrm{ETCO}_{2}$ was not sufficient in patients with respiratory distress or during spontaneous breathing [17]. According to our results, when using a method assuming a stable gap between $\mathrm{ETCO}_{2}$ and $\mathrm{PaCO}_{2}$, the value of $\mathrm{PaCO}_{2}$ is underestimated, which might lead to insufficient ventilation. Probably, the gradient between $\mathrm{ETCO}_{2}$ and $\mathrm{PaCO}_{2}$ does not remain constant when a patient's clinical condition changes; this decreases the accuracy. Belenkiy and colleagues used volumetric capnography (Vcap) in a porcine model of chest trauma and showed that the difference between the estimated and measured $\mathrm{PaCO}_{2}$ values changed only slightly [18].

Transcutaneous $\mathrm{CO}_{2}$ (tc- $\mathrm{CO}_{2}$ ) monitoring has been used especially in neonates and infants [19]. The accuracy of tc- $\mathrm{CO}_{2}$ has varied in studies and, for example, during low flow shock, tc- $\mathrm{CO}_{2}$ is not a consistently accurate reflection of $\mathrm{PaCO}_{2}$ [20] and is dependent on microvascular circulation [21]. Estimations based on end-tidal carbon dioxide may be sufficient in selected patients, assuming the clinical condition is stable, which is not the case in many critically ill patients in the ICU or prehospital setting. 
In this study, the interventions undertaken to influence $\mathrm{CO}_{2}$ in a few cases caused cardiovascular and respiratory collapse. As expected and due to the rapid decrease in cardiac output (CO) and lung perfusion, this resulted in much poorer accuracy of both formulae estimating $\mathrm{PaCO}_{2}$. Ornato et al. showed that the relationship between $\mathrm{CO}$ and $\mathrm{ETCO}_{2}$ was more logarithmic than linear, resulting in big changes in $\mathrm{ETCO}_{2}$ when changes in $\mathrm{CO}$ were rather small [22]. During low or high $\mathrm{CO}, \mathrm{ETCO}_{2}$ seems to be a poor indicator of $\mathrm{PaCO}_{2}$. In prehospital settings, $\mathrm{ETCO}_{2}$ cannot estimate $\mathrm{PaCO}_{2}$, since the gradient between $\mathrm{PaCO}_{2}$ and $\mathrm{ETCO}_{2}$ varies greatly. The difference is especially notable in patients with hypocapnia [23]. Similar results have been shown with other methods using $\mathrm{ETCO}_{2}$ for the estimation of $\mathrm{PaCO}_{2}$. Mechanically ventilated patients with severe trauma or burns have poorer correlation between $\mathrm{ETCO}_{2}$ and $\mathrm{PaCO}_{2}$ in prehospital settings, with acidosis and poor outcome as consequences [24]. When $\mathrm{FIO}_{2}$ approaches $100 \%$, the reliability of the novel formula is impaired. In one case during our study, a tube occlusion problem occurred, and the difference between $\mathrm{PaCO}_{2}$ and calculated $\mathrm{PaCO}_{2}$ was near $5 \mathrm{kPa}$. In addition to the obvious unreliability of $\mathrm{ETCO}_{2}$ associated with tube occlusion, the high difference was partly due to high $\mathrm{FIO}_{2}$, as pulse oximetry is a poor indicator of $\mathrm{PaO}_{2}$ [25]. Indeed, our results are preliminary and cannot be generalized at this stage to other populations. In an ongoing further study, our aim is to test the accuracy of the presented formula with a large group of mechanically ventilated ICU patients with and without respiratory failure.

If this novel formula is validated in clinical studies, a built-in algorithm in patient monitoring could offer continuous information about a patient's estimated $\mathrm{PaCO}_{2}$ level. This could be valuable in settings without the possibility of ABG analysis. Indeed, our results show that the novel algorithm was more accurate than estimating carbon dioxide with end-tidal $\mathrm{CO}_{2}$, which is commonly used in the prehospital setting. Eventually, ventilators that keep patients' $\mathrm{CO}_{2}$ levels stable by modifying tidal volume and pressure may be developed; using an equation embedded in the ventilator for estimating arterial $\mathrm{CO}_{2}$ tension is one possible practical application.

Our study has some important limitations. First, the number of individual animals subjected to different experiments was small and, thus, the respiratory and hemodynamic conditions were not standardized. Second, ABG analyses were not taken at specified time points, and the number of ABG analyses varied. Third, as mentioned previously, remarkable changes in cardiac output result in a poor capacity to estimate $\mathrm{PaCO}_{2}$ with the traditional method. In this study we did not measure cardiac output; hence, it is unclear to what extent it applies to the new algorithm. Fourth, we did not use hyperventilation or very high $\mathrm{FIO}_{2}$ in the experiments. At this point, it is uncertain how the new algorithm functions for patients with hypocapnia or in situations where a high $\mathrm{FIO}_{2}$ is used. In the setup of the original study, the goal was normoventilation while manipulating $\mathrm{PaCO}_{2}$ in various interventions. In addition, the determination of factor $\mathrm{k}$ used in the algorithm may change with more datapoints. In situations where $\mathrm{ETO}_{2}$ is remarkably high, for example, massive shunting in lungs, the algorithm does not function. Finally, despite differences in the oxygen dissociation curve between humans and pigs, we did not use a porcine-specific method for measuring hemoglobin saturation, possibly overestimating the $\mathrm{PaO}_{2}$ level [25]. Finally, the samples were obtained in animals ventilated with a novel adaptive ventilation control system. However, this novel ventilation system uses common ventilation modes that are not likely to influence the end-tidal concentrations of either oxygen or carbon dioxide any differently than other currently used mechanical ventilators. Nonetheless, the inherent limitations of the study design are not likely to overestimate the accuracy of the novel algorithm, but the opposite. Some of the limitations, such as lack of standardization of the experiments, render the study sample more similar to a clinical real-life scenario than a tightly controlled experimental setting would have done.

\section{Conclusions}

We present a new formula for estimating $\mathrm{PaCO}_{2}$ noninvasively using pulse oximetry and measurement of inspired and expired gas fractions of $\mathrm{CO}_{2}$ and $\mathrm{O}_{2}$. In this preliminary animal study, 
this formula appears to have a reasonable agreement with $\mathrm{PaCO}_{2}$ values measured with $\mathrm{ABG}$ analysis, but it needs further validation in human patients.

Supplementary Materials: The following are available online at http:/ /www.mdpi.com/2077-0383/7/9/290/s1, Figure S1: The flowchart of experimental protocol, Figure S2a: The Bland-Altman plot demonstrating the difference between estimated $\mathrm{PaCO}_{2}$ (Formula 1) and measured $\mathrm{PaCO}_{2}$ with 95\% confidence intervals in animals 9-19, Figure S2b: The Bland-Altman plot demonstrating the difference between estimated $\mathrm{PaCO}_{2}$ (Formula 2) and measured $\mathrm{PaCO}_{2}$ with 95\% confidence intervals in animals 9-19, Figure S3: The differences between measured $\mathrm{PaCO}_{2}$ and end-tidal $\mathrm{CO}_{2}$ values at each time point, Figure S4: The Bland-Altman plot demonstrating the difference between measured $\mathrm{PaCO}_{2}$ and end-tidal carbon dioxide $\left(\mathrm{ETCO}_{2}\right)$ with $95 \%$ confidence intervals, Figure S5a: The Bland-Altman plot demonstrating the difference between estimated $\mathrm{PaCO}_{2}$ (Formula 1) and $\mathrm{paCO}_{2}$ with $95 \%$ confidence intervals in 26 cases prior to any intervention aiming at modifying $\mathrm{CO}_{2}$, Figure $\mathrm{S} 5 \mathrm{~b}$ : The Bland-Altman plot demonstrating the difference between end-tidal carbon dioxide $\left(\mathrm{ETCO}_{2}\right)$ and $\mathrm{paCO}_{2}$ with $95 \%$ confidence intervals in 26 cases prior to any intervention aiming at modifying $\mathrm{CO}_{2}$, Table S1: Weight, interventions performed, number of $\mathrm{ABG}$ analyses, values of measured $\mathrm{PaCO}_{2}$ and $\mathrm{pH}$ values in the study subjects. The numbering of animals are based on timing, with the first experimental animal labelled number one and the last number nineteen, Table S2: Hemodynamic variables, etCO $\mathrm{CtO}_{2}, \mathrm{PaO}_{2}$ and $\mathrm{PaO}_{2} / \mathrm{FiO}_{2}$ ratios of each animal during the experiment, Table S3: Estimation of $\mathrm{PaO}_{2}$ based on values of oxygen-dissociation curve.

Author Contributions: R.R., E.H., P.H.R., T.H. and M.B.S. conceived the study, its concept, and its design. R.R., J.H., E.H. and M.B.S. had full access to all the data and take responsibility for data integrity and accuracy of the analysis. R.R., J.H. and M.B.S. drafted the manuscript. All authors read and contributed to the manuscript in its final form.

Funding: GE Healthcare was the study sponsor for the animal experiments that were undertaken to test a novel mechanical ventilator. The current analysis was not part of the original protocol.

Acknowledgments: This study was financed with unrestricted funding from Helsinki University (Three-year research grant 2016-2018, H3702-11-103568, wbs 73702705) and Helsinki University Hospital (State funding, VTR-Y102011094), and research grants from Finska Läkaresällskapet. The authors also wish to thank the staff of the Research and Development Unit of Helsinki University Hospital, i.e., Veikko Huusko and Olli Valtanen.

Conflicts of Interest: Authors Erkki Heinonen and Tom Häggblom are employees of GE Healthcare. Markus Skrifvars has received research funding from GE Healthcare and lecture fees from Covidien, Orion Pharma, Axis Shield Diagnostics and BARD. All other authors report no conflicts of interest.

\section{References}

1. Slutsky, A.S. Mechanical ventilation. American College of Chest Physicians' Consensus Conference. Chest 1993, 104, 1833-1859. [CrossRef] [PubMed]

2. Curley, G.; Laffey, J.G.; Kavanagh, B.P. Bench-to-bedside review: Carbon dioxide. Crit. Care 2010, 14, 220. [CrossRef] [PubMed]

3. Marhong, J.; Fan, E. Carbon dioxide in the critically ill: Too much or too little of a good thing? Respir. Care 2014, 59, 1597-1605. [CrossRef] [PubMed]

4. Melanson, S.E.; Szymanski, T.; Rogers, S.O.; Jarolim, P.; Frendl, G.; Rawn, J.D.; Cooper, Z.; Ferrigno, M. Utilization of arterial blood gas measurements in a large tertiary care hospital. Am. J. Clin. Pathol. 2007, 127, 604-609. [CrossRef] [PubMed]

5. Ghajar, J. Traumatic brain injury. Lancet 2000, 356, 923-929. [CrossRef]

6. Falkenbach, P.; Kamarainen, A.; Makela, A.; Kurola, J.; Varpula, T.; Ala-Kokko, T.; Perttila, J.; Tenhunen, J. Incidence of iatrogenic dyscarbia during mild therapeutic hypothermia after successful resuscitation from out-of-hospital cardiac arrest. Resuscitation 2009, 80, 990-993. [CrossRef] [PubMed]

7. Al-Otaibi, H.M.; Hardman, J.G. Prediction of arterial oxygen partial pressure after changes in $\mathrm{FIO}(2)$ : Validation and clinical application of a novel formula. Br. J. Anaesth. 2011, 107, 806-812. [CrossRef] [PubMed]

8. Vaahersalo, J.; Bendel, S.; Reinikainen, M.; Kurola, J.; Tiainen, M.; Raj, R.; Pettila, V.; Varpula, T.; Skrifvars, M.B.; Finnresusci Study Group. Arterial blood gas tensions after resuscitation from out-of-hospital cardiac arrest: Associations with long-term neurologic outcome. Crit. Care Med. 2014, 42, 1463-1470. [CrossRef] [PubMed]

9. Helm, M.; Hauke, J.; Lampl, L. A prospective study of the quality of pre-hospital emergency ventilation in patients with severe head injury. Br. J. Anaesth. 2002, 88, 345-349. [CrossRef] [PubMed] 
10. West, E.; Bardell, D.; Senior, J.M. Comparison of the EPOC and i-STAT analysers for canine blood gas and electrolyte analysis. J. Small Anim. Pract. 2014, 55, 139-144. [CrossRef] [PubMed]

11. Maynard, R. Nunn's Applied Respiratory Physiology, 5th ed.; Occup Environ Med: London, UK, 2000.

12. Serianni, R.; Barash, J.; Bentley, T.; Sharma, P.; Fontana, J.L.; Via, D.; Duhm, J.; Bunger, R.; Mongan, P.D. Porcine-specific hemoglobin saturation measurements. J. Appl. Physiol. 2003, 94, 561-566. [CrossRef] [PubMed]

13. Bland, J.M.; Altman, D.G. Statistical methods for assessing agreement between two methods of clinical measurement. Lancet 1986, 1, 307-310. [CrossRef]

14. Olofsen, E.; Dahan, A.; Borsboom, G.; Drummond, G. Improvements in the application and reporting of advanced Bland-Altman methods of comparison. J. Clin. Monit. Comput. 2015, 29, 127-139. [CrossRef] [PubMed]

15. Bendjelid, K.; Schutz, N.; Stotz, M.; Gerard, I.; Suter, P.M.; Romand, J.A. Transcutaneous $\mathrm{PCO}_{2}$ monitoring in critically ill adults: Clinical evaluation of a new sensor. Crit. Care Med. 2005, 33, 2203-2206. [CrossRef] [PubMed]

16. Razi, E.; Moosavi, G.A.; Omidi, K.; Khakpour Saebi, A.; Razi, A. Correlation of end-tidal carbon dioxide with arterial carbon dioxide in mechanically ventilated patients. Arch. Trauma Res. 2012, 1, 58-62. [CrossRef] [PubMed]

17. Kerr, M.E.; Zempsky, J.; Sereika, S.; Orndoff, P.; Rudy, E.B. Relationship between arterial carbon dioxide and end-tidal carbon dioxide in mechanically ventilated adults with severe head trauma. Crit. Care Med. 1996, 24, 785-790. [CrossRef] [PubMed]

18. Belenkiy, S.M.; Baker, W.L.; Batchinsky, A.I.; Mittal, S.; Watkins, T.; Salinas, J.; Cancio, L.C. Multivariate analysis of the volumetric capnograph for $\mathrm{PaCO}_{2}$ estimation. Int. J. Burns Trauma 2015, 5, 66-74. [PubMed]

19. Binder, N.; Atherton, H.; Thorkelsson, T.; Hoath, S.B. Measurement of transcutaneous carbon dioxide in low birthweight infants during the first two weeks of life. Am. J. Perinatol. 1994, 11, 237-241. [CrossRef] [PubMed]

20. Tremper, K.K.; Shoemaker, W.C.; Shippy, C.R.; Nolan, L.S. Transcutaneous $\mathrm{PCO}_{2}$ monitoring on adult patients in the ICU and the operating room. Crit. Care Med. 1981, 9, 752-755. [CrossRef] [PubMed]

21. Spelten, O.; Fiedler, F.; Schier, R.; Wolfgang, A.; Wetsch Hinkelbein, J. Transcutaneous $\mathrm{PTCCO}_{2}$ measurement in combination with arterial blood gas analysis provides superior accuracy and reliability in ICU patients. J. Clin. Monit. Comput. 2017, 31, 153-158. [CrossRef] [PubMed]

22. Ornato, J.P.; Garnett, A.R.; Glausen, F.L. Relationship between cardiac output and the end-tidal carbon dioxide tension. Ann. Emerg. Med. 1990, 19, 1104-1106. [CrossRef]

23. Belpomme, V.; Ricard-Hibon, A.; Devoir, C.; Dileseigres, S.; Devaud, M.L.; Chollet, C.; Marty, J. Correlation of arterial $\mathrm{PCO}_{2}$ and $\mathrm{PETCO}_{2}$ in prehospital controlled ventilation. Am. J. Emerg. Med. 2005, 23, 852-859. [CrossRef] [PubMed]

24. Cooper, C.J.; Kraatz, J.J.; Kubiak, D.S.; Kessel, J.W.; Barnes, S.L. Utility of Prehospital Quantitative End Tidal $\mathrm{CO}_{2}$ ? Prehosp. Disaster Med. 2013, 28, 87-93. [CrossRef] [PubMed]

25. Tusman, G.; Bohm, S.H.; Suarez-Sipmann, F. Advanced Uses of Pulse Oximetry for Monitoring Mechanically Ventilated Patients. Anesth. Analg. 2017, 124, 62-71. [CrossRef] [PubMed]

(C) 2018 by the authors. Licensee MDPI, Basel, Switzerland. This article is an open access article distributed under the terms and conditions of the Creative Commons Attribution (CC BY) license (http://creativecommons.org/licenses/by/4.0/). 